\title{
Intraoperative Manufacturing of Patient Specific Instrumentation for Shoulder Arthroplasty: a Novel Mechatronic Approach
}

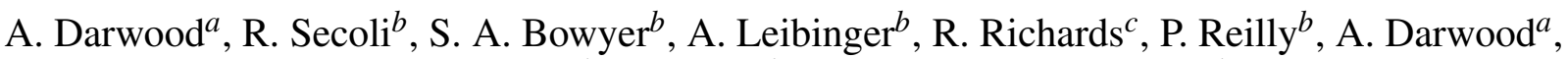 \\ A. Tambe ${ }^{d}$, R. Emery ${ }^{b}$ and F. Rodriguez y Baena ${ }^{b}$ \\ ${ }^{a}$ Prometheus Surgical Ltd. 24 Upper Wimpole St, London, United Kingdom. \\ email: alastairdarwood@hotmail.com \\ ${ }^{b}$ Imperial College, London, United Kingdom \\ c 3Cavendish Implants, London, United Kingdom \\ ${ }^{d}$ Derby Hospital NHS Trust, London, United Kingdom
}

\begin{abstract}
Optimal orthopaedic implant placement is a major contributing factor to the long term success of all common joint arthroplasty procedures. Devices such as three-dimensional (3D) printed, bespoke guides and orthopaedic robots are extensively described in the literature and have been shown to enhance prosthesis placement accuracy. These technologies, however, have significant drawbacks, such as logistical and temporal inefficiency, high cost, cumbersome nature and difficult theatre integration. A new technology for the rapid intraoperative production of patient specific instrumentation, which overcomes many of the disadvantages of existing technologies, is presented here. The technology comprises a reusable table side machine, bespoke software and a disposable element comprising a region of standard geometry and a body of mouldable material. Anatomical data from Computed Tomography (CT) scans of 10 human scapulae was collected and, in each case, the optimal glenoid guidewire position was digitally planned and recorded. The achieved accuracy compared to the preoperative bespoke plan was measured in all glenoids, from both a conventional group and a guided group. The technology was successfully able to intraoperatively produce sterile, patient specific guides according to a pre-operative plan in 5 minutes, with no additional manufacturing required prior to surgery. Additionally, the average guide wire placement accuracy was $1.58 \mathrm{~mm}$ and $6.82^{\circ}$ degrees in the manual group, and $0.55 \mathrm{~mm}$ and $1.76^{\circ}$ degrees in the guided group, also demonstrating a statistically significant improvement.
\end{abstract}

Keywords: Arthroplasty; Computer Assisted Surgery; Medical Robotics

\section{Introduction}

It is well known that the accurate placement of orthopaedic hardware, such as arthroplasty prostheses, into a biomechanically derived optimum position is of paramount importance for the long term success of these interventions. ${ }^{1-3}$ Arthroplasty prosthesis malpositioning has been shown to decrease postoperative function and implant longevity in many of the major joints. For example, De Haan et al. ${ }^{3}$ show acetabular component malpositioning to be a significant risk factor for revision hip arthroplasty, whilst Skirving et al. ${ }^{4}$ similarly show poor glenoid component positioning to be a major reason for arthroplasty failure and subsequent revision. Choong et al. ${ }^{2}$ showed that improved accuracy with respect to the anatomical alignment of implanted knee prostheses resulted in an improvement of $19 \%$ in the post-operative international knee score. Similarly, in many orthopaedic procedures, it is advantageous or even essential to know the exact placement of hardware such as screws or pins, for example in spinal surgery, complex fracture reduction, or the position of osteotomy cuts.

The need for orthopaedic guidance has resulted in significant growth in technologies able to assist a surgeon in the placement of hardware or tools, with many companies marketing a number of guidance products to consumers. Guidance technologies may be broadly split into three main categories: bespoke patient specific instrumentation, such as rapid manufactured (3D printed) guides, Computer Aided Surgery (CAS) systems, and intraoperative orthopaedic surgical robots.

Bespoke 3D printed guides are commercially available from many large orthopaedic implant manufacturers and standalone companies. Guides are digitally designed from manipulated Computed Tomography (CT) or Magnetic Resonance Imaging (MRI) data of a patient, and subsequently 3D printed, sterilised and sent to a surgeon for intraoperative use. Guides 
comprise at least one surface topographically matching an area of patient osseous anatomy, allowing placement onto a preplanned site, and tool guidance channels to facilitate osseous modification according to a pre-operative plan. ${ }^{5}$ Despite relatively wide availability, bespoke patient guides have a number of well recognised drawbacks. Guides are typically costly, their production involves inefficient logistical chains and their imaging data based geometry occasionally does not lend itself to fitting physical patient anatomy at the time of surgery, risking inaccuracy. Digital plans for guides must be sent between a surgeon and a company engineer before final physical manufacture of any guide. Following manufacture, the guide must be cleaned and processed before packaging and shipping to the surgeon at some point before the procedure.

CAS systems provide intraoperative visualization of the anatomy and enable tracking of the limb and surgical tools during the arthroplasty procedure. They do not, however, provide any active assistance in terms of instrument placement or bone machining. This is entirely driven by the ability of the surgeon to follow visual cues, as provided by a graphical software front end. Additionally, the high cost and system footprint, which adds to an already cluttered OR environment, have been resisted by surgeons, possibly explaining the limited uptake of this technology in orthopedics.

Intraoperative surgical robots have recently undergone significant development, with commercial technologies such as Stryker's Mako Rio (c) and Blue Belt's C Navio c) receiving much surgeon attention. In 2003, Cobb et al. demonstrated good preliminary clinical data with the Acrobot Technology, ${ }^{6}$ showing how a robot might be a useful tool for arthroplasty procedures. ${ }^{7}$ Whilst orthopaedic robotics provides an alternative approach to hardware guidance, it too suffers from a number of drawbacks, such as a very high capital cost, long set up and cleaning times, large physical size, and generally an increased cost per operation. ${ }^{8,9}$ It should be noted that small scale, patient mounted robots such as the Mazor Renaissance are more convenient in terms of theatre integration, but suffer from many similar drawbacks as larger devices.

The authors have found few past works related to the intraoperative production of surgical guides, perhaps due to the difficulty of deploying rapid manufacturing processes within the operating theatre. In similar work, implants have been produced intraoperatively - in 1992, Salvi ${ }^{10}$ describes results from the now discontinued Identifit system for the intraoperative manufacture of a patient specific femoral stem from an intraoperative mould of the femoral canal. The mould was taken to a nearby Computer Numerical Control (CNC) suite, where a laser scan was taken and the surface data used by a separate CNC milling machine to produce a custom implant stem. This stem was sterilised and returned to theatre to be implanted into the patient, with a total manufacture time of 40 minutes.

Considering the established importance of orthopaedic guidance, this paper presents a new technology developed to overcome many of the drawbacks of existing approaches, whilst providing a means to accurately place orthopaedic hardware according to a pre-operative plan. Work focussed on a low-cost implementation, which also removes the logistical chain of 3D printed bespoke guides and complete reliance on preoperative patient imaging data.

The approach presented here allows accurate bespoke drill/cutting guides to be rapidly produced in the operating theatre itself, by employing a simple, low cost hardware element and disposable consumables. In this paper, the technology is described in the context of total shoulder arthroplasty, more specifically, the placement of the glenoid component according to a pre-operative plan.

The paper is structured as follows: Section 2 reports on the description of the mechatronic system, with focus on the mechanical design and the required disposable consumables. Section 3 and Section 4 describe the procedures followed during a clinical proof of concept trial to successfully place the glenoid component, and the required (pre- and intra-operative) control software, respectively. Section 5 describes the results of a set of laboratory trials performed using this new technology. The paper concludes with a discussion section and conclusion.

\section{Mechatronic Device}

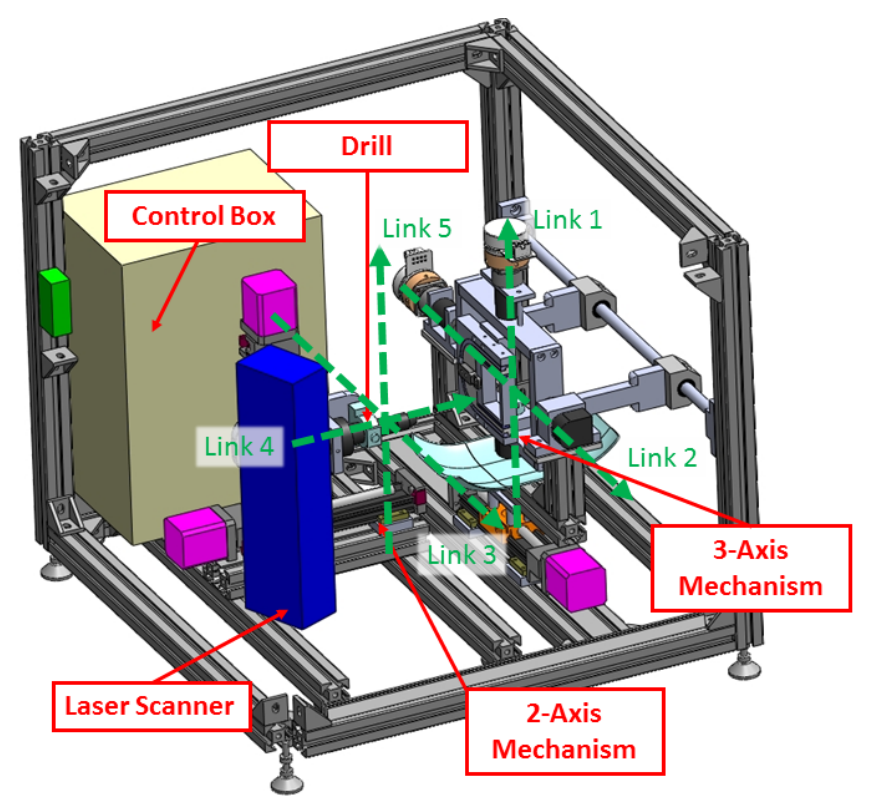

Fig. 1. Render of the mechatronic system. Kinematic link axes corresponding to the $\mathrm{DH}$ parameters in table 1 are also shown here.

\subsection{Mechatronic Hardware}

A five Degrees Of Freedom (DOF) mechatronic system was developed for the patient specific placement of drill holes by means of an automated, off-the-shelve drill (RS 12V-max 16000rpm drill), which is used to machine a hole within a disposable element. The mechatronic system, which is rendered in Fig. 1, is formed by two separate subsystems.

The first subsystem is a mechanism with 2 DOF holding the drill. It is assembled from two linear stages (Velmex XSlide - lead-screw pitch $1 \mathrm{~mm} / \mathrm{rev}$, stroke $150 \mathrm{~mm}$ ) and controlled by 
stepper motors (ARCUS-DMX-A2-Dr Nema 17) with position feedback of 1000 pulses/rev, resulting in an overall accuracy of $1 / 4000 \mathrm{~mm}$.

The second mechanism, shown in Fig. 2, has 3 additional DOF (two revolute and one linear). The rotational DOF are driven by backlash-free harmonic gearboxes (CSF-8-100-2XH-J Harmonic Drive AG, Germany) connected to compact DC Motors (MAXON EC32-flat). Feedback encoders (AVAGO HEDR$3600-3600$ pulse/rev), mounted on the inner and outer shafts of the rotational axes, provide an angular resolution of $70 \mu^{\circ}$. The two rotational plates are translated by a linear stage (Velmex XSlide - leadscrew pitch $1 \mathrm{~mm} / \mathrm{rev}$, stroke $100 \mathrm{~mm}$ ) driven by a stepper motor (ARCUS-DMX-A2-Dr Nema 23) with position feedback of 1000 pulse/rev, resulting in a overall accuracy matching the first mechanism.

The five-link kinematic structure for the complete device, with nominal positional accuracy of $0.25 \mu \mathrm{m}$ for the linear stages and $70 \mu^{\circ}$ for the rotational stages, is shown in Fig. 1 and the associated Denavit-Hartenberg (DH) parameters are shown in Table 1. Three controllers were employed to drive the $5 \mathrm{DOF}$ assembly: one ARCUS PMX-4EX-SA to control the linear stages and two Maxon EPOS2 24/2, used to control the two rotational stages. For safety purposes, a bespoke safety control board was designed to function as a watchdog for hardware malfunctions, such as jamming of the actuators, misuse of the operator or the activation of emergency stop buttons.

Table 1. Nominal DH parameters for the robot design. Parameters go from the gimbal centre (link 0) to the drill (link 5).

\begin{tabular}{ccccc}
\hline Link & $d_{i}$ & $\theta_{i}$ & $a_{i}$ & $\alpha_{i}$ \\
\hline 1 & 0 & $\theta_{1}$ & 0 & $\pi / 2$ \\
2 & 0 & $\theta_{2}$ & -25 & 0 \\
3 & $d_{3}$ & $\pi$ & 0 & $\pi / 2$ \\
4 & $d_{4}$ & $-\pi / 2$ & 0 & $\pi / 2$ \\
5 & $d_{5}$ & 0 & 0 & 0 \\
\hline
\end{tabular}

The total reachable workspace of the machine is approximately $1,000 \mathrm{~cm}^{3}$, defined as the cubic volume laying on the surface of the inner rotational plate when orthogonal to link 4 , as shown in Fig. 2. The inner rotational plate has a base of $100 \times 100 \mathrm{~mm}$, and is able to rotate more than $30^{\circ}$ around the principal rotational axis.

A commercial structured-light 3D scanner (HDI 120 Blue Led - LMI3D Tech.), with a resolution up to 60 microns (depending on imaging parameters and lighting conditions), was fixed inside the robot to acquire surface images of the mouldable component.

The hardware cost totalled $£ 17,377$, comprising predominantly off the shelf components, with a small number of bespoke $\mathrm{CNC}$ milled parts, with the potential for further cost reductions through mass production.

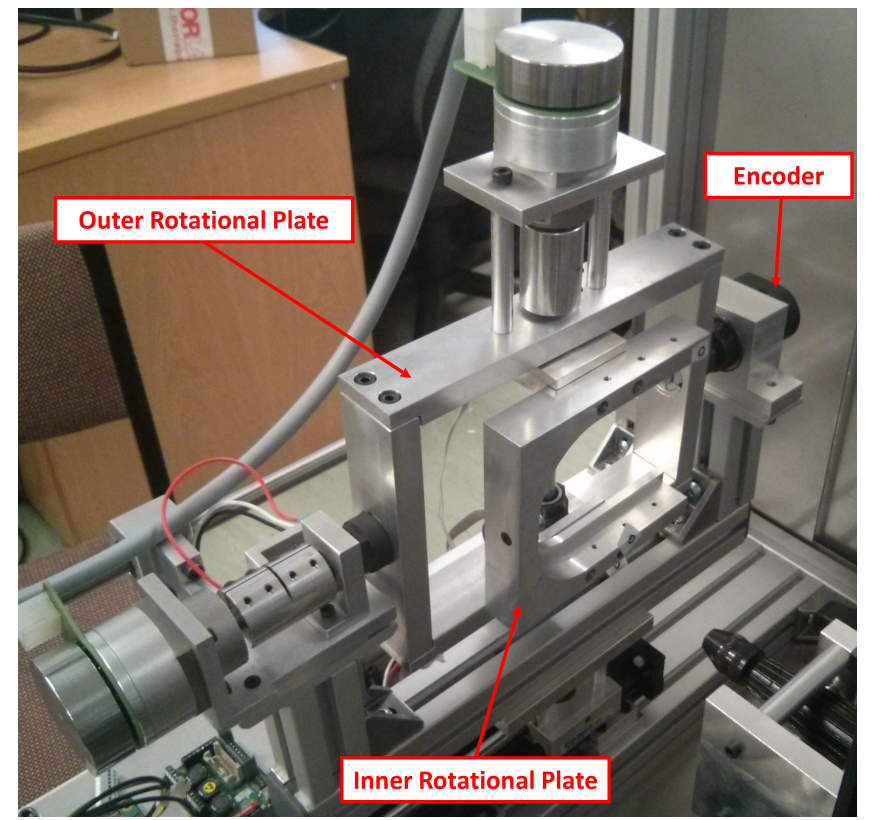

Fig. 2. View of the 3-axes subsystem ('the Gimbal'):the inner plate is free to rotate without constraints in the range of motion, while the outer plate is constrained, with an angular inclination in the range of $\approx \pm 30^{\circ}$

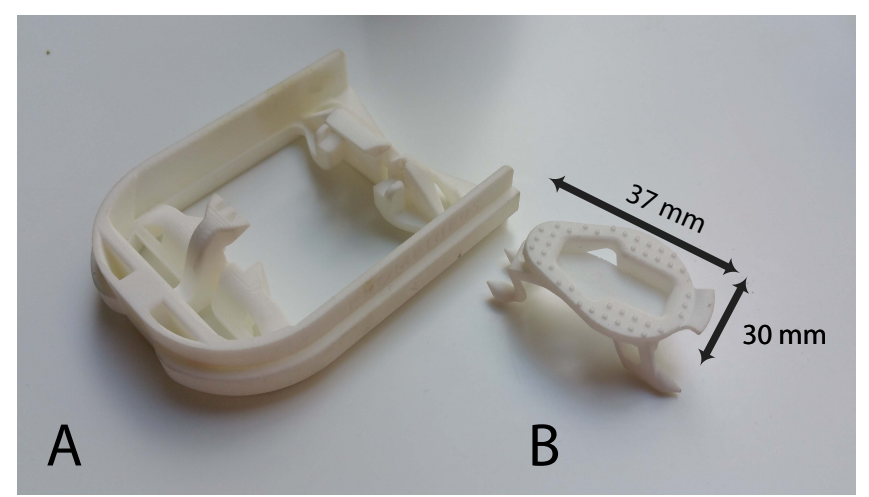

Fig. 3. A: disposable socket to be placed within the system. B: disposable socket for the mould

\subsection{Disposable Components}

The sterile disposables comprise of a plastic element (in ABS, rapid prototyped plastic) with standard geometry, coated on one side with a body of mouldable material (Vinyl Polysiloxane EXABITE II ${ }^{\mathrm{TM}}$ GC America Inc.) and a carrier element, which provides two degrees of separation between the sterile component and the non-sterile surfaces of the Inner Rotational Plate within the gimbal of the mechatronic system. The disposable element is provided in a configuration relating to the anatomical area of use. In this work, the disposable component shown in 


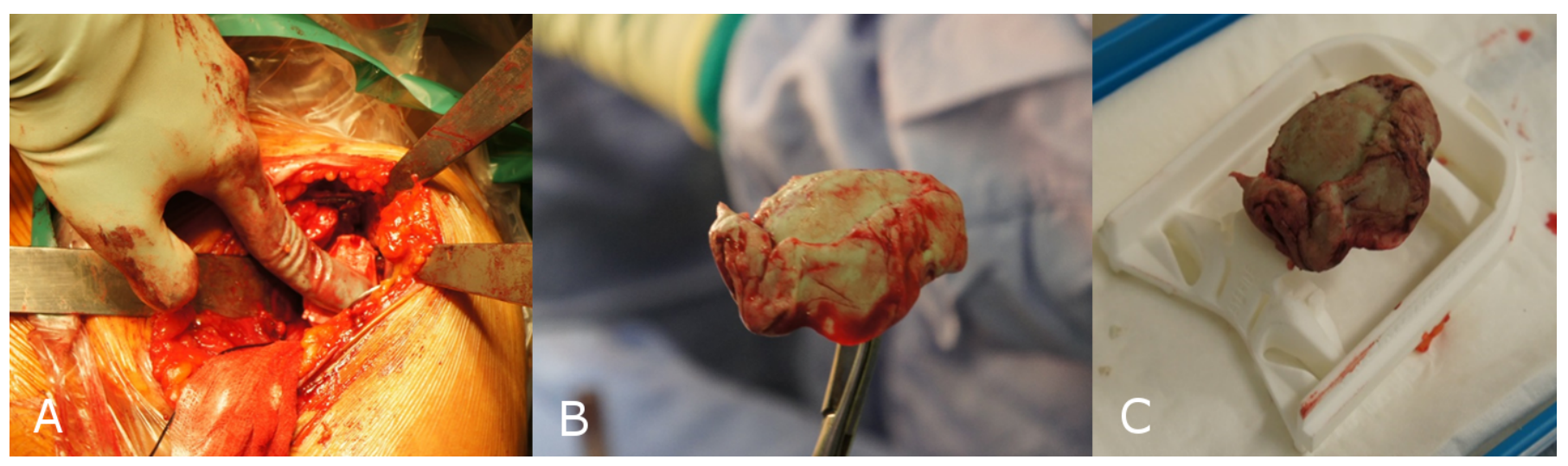

Fig. 4. Procedure step (3) ${ }^{\mathrm{a}}$ : insertion of the sterile disposable mould into the glenoid cavity (A). Once the mould has been pressed into the glenoid surface, it is removed (B) and placed in the corresponding receptor apparatus (C).

Fig. 3B is provided in a configuration fitting within the glenoid cavity of a patient. The components are provided in multiple sizes. The disposable component is inserted into the receptor assembly (the inner rotational plate) in a pre-defined manner, by virtue of its standard geometry, and is rigidly held in place, as shown in Fig. 3A-B. The cost of conventional three dimensional printed guides, excluding the required imaging study needed to produce a patient-specific plan (Section 4), varies in the literature between $\$ 700-\$ 1500,{ }^{11,12}$ since these are bespoke and are manufactured on a per-patient basis. In comparison, the described disposable consumables in this approach come in a standard shape set and can thus be mass produced and sterilised in large volumes. It is envisaged the consumable may be manufactured with a rapid setting mouldable material already in place, but, for the purposes of this paper, sample disposable consumables were $3 \mathrm{D}$ printed in nylon as a small batch (Eos Formiga SLS printer). Disposable components were produced at a cost price of $£ 1.50$ each with an additional $£ 2$ of mouldable material. Each consumable was subsequently steam sterilised in a medical facility, resulting in an approximate cost per patient of disposable consumables of $£ 15$ per patient.

\section{Intraoperative Workflow}

The procedural workflow to perform the robotic-assisted arthoplasty is described as follows:

(1) Planning is carried out on CT or MRI patient data. This process defines the guide wire position as a vector within the coordinate frame of reference of the digitally reconstructed, patient specific scapula model. This plan may be carried out pre-operatively or intraoperatively, but prior to any bone machining.

(2) The surgeon exposes the surgical site in the conventional manner. In this case, exposing the osseous anatomy of the glenoid cavity.
(3) The sterile disposable consumable is prepared. The mouldable side of the component is pressed into the surgical site, as in Fig. 4A (the glenoid cavity), which quickly solidifies, forming a mould of the glenoid cavity and exposed portions of its rim. The component is removed (Fig. 4B) and placed within the sterile carrier (Fig. 4C), which in turn is placed into the corresponding receptor assembly of the mechatronic device.

(4) The optical scanner is used to digitise the moulded surface and an automatic registration routine is then initiated to match the digitised mould surface to the imaging data derived from segmented, patient specific MR or CT data. In this way, the scanned glenoid surface model is automatically placed into the same coordinate frame of reference as the original 3D patient scapula data. As a result, the vector describing the location of the guide wire may now intersect the digital glenoid surface mould model.

(5) From this data, a suitable drilling path is now created interactively by the user. The drill, fitted with a sterile drill bit, is repositioned according to the output of a planning module (Section 4), which reproduces the intended drill path in an appropriate coordinate system. Fig. 5 shows the final stage of this step.

(6) The sterile moulded component is removed from the machine and returned to the operative site. The part is held onto the anatomy with slight external pressure from either the surgeon or assistant. Its solidified moulded geometry rigidly constrains it to the near-identical position from where the mould was taken. The prepared guide hole is now drilled through by the surgeon in the conventional way, enabling placement of a guide wire into the pre-planned position. Once this is complete, the disposable is slid off the guide wire and discarded, leaving the wire in the preoperatively planned position. The surgery continues in the conventional manner.

${ }^{a}$ Full informed consent was obtained for the patient undergoing this procedure to participate in this study. 


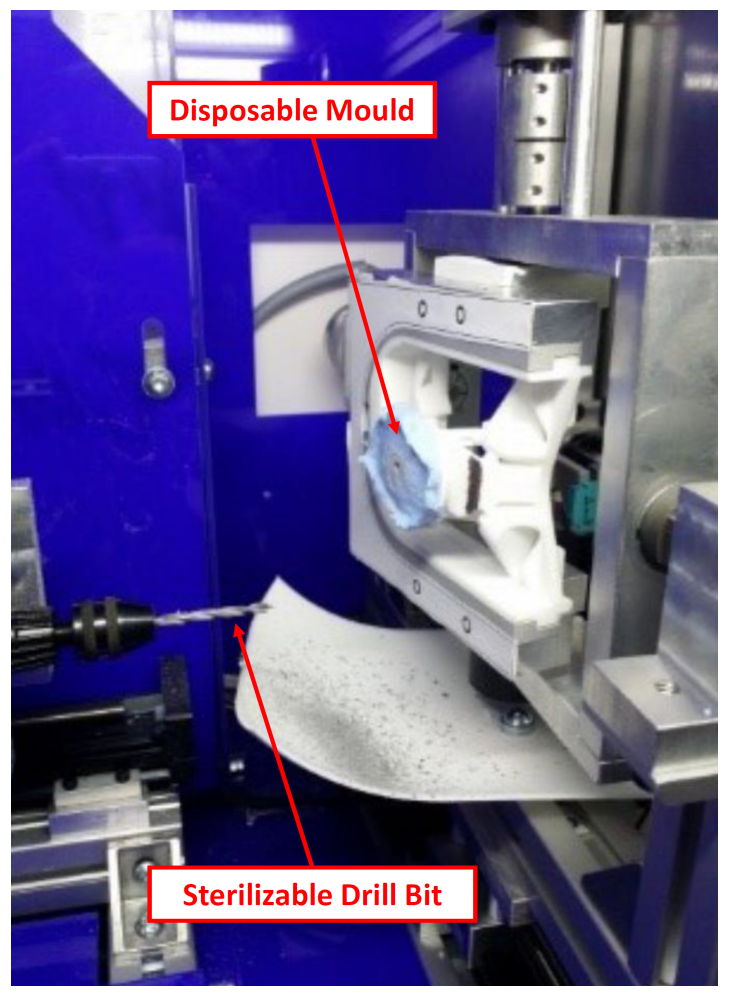

Fig. 5. View of the disposable component after the creation of the guide (intraoperative workflow step 5)

\section{Software}

This section describes the software to perform the registration between the glenoid mould and the CT scan, as well as the software to generate the motion plan to machine the mould according to a preoperatively defined configuration. The overall structure of the software's workflow, and these modules' interaction, is shown in Fig. 6.

\subsection{Planning Software}

A planning module was created and incorporated into the device control software workflow. In this instance, 'planning' is defined as the specification of a desired axis within the coordinate frame of reference of a 3D digital rendering of patient anatomy (a scapula CT scan in the case of this paper). This axis describes the intended placement of a guidewire into the patient anatomy, as to correctly position an arthroplasty prosthesis. A user imports a stereolithography (STL) file comprising segmented patient anatomy derived from imaging data. An operator can then manually define the desired orientation and position of one or more virtual guide wires within the frame of reference of the 3D rendering of patient anatomy (as derived from imaging data) by selecting medial and lateral axis intersection points. Once speci- fied, the desired axis is subsequently saved within the coordinate frame of reference of the digital anatomy reconstruction.

Such a simple method to describe the intended axis orientation is likely unsuitable for a future medical product, as many manufactures of orthopaedic implants already produce a multitude of digital planning tools to plan the ideal position of a virtual prosthesis prior to surgery. However, this study focused on the accuracy with which a digital plan could be physically re-created, regardless of whether or not the plan was clinically optimum. In future device iterations, the planning module will be substituted by fully functional surgical planning software.

\subsection{Optical Kinematic Calibration}

To maximise the accuracy of drill hole placement within the mould, under the achievable manufacturing tolerances, an optical kinematic calibration of the robot was performed after initial construction and whenever the system was packaged and transported. The mechanical design and manufacturing process minimised the misalignment between the drill axis and the linear stage responsible for drill insertion. This meant that it was possible to use nominal DH parameters for the final link in the kinematic chain, and drill the hole through the mould by advancing a single linear actuator. However, the remaining DH parameters (nominal values for which are given in Table 1) were calibrated.

The calibration was performed using a custom 3-marker rigid body, which defined a unique $3 \mathrm{D}$ coordinate frame, and the 3D scanner used for the mould geometry acquisition. The plane and axis of each robot link were identified by placing the custom 3-marker rigid body in the chuck of the drill and then moving each degree of freedom of the robot sequentially. A series of images were taken with the scanner in different robot configurations, from which the 6-D Cartesian poses of each end of the robot could be accurately computed. Based on the calibration method described by Abderrahim and Whittaker, ${ }^{13}$ the end effector poses and respective joint configurations were used to accurately compute the angle and offset of each kinematic link in the robot, giving the full DH parameters. For the prototype device, the calibrated parameter residuals were all smaller than 0.5 $\mathrm{mm} / 0.5^{\circ}$, demonstrating the reliability of the robot's manufacture. As the calibration was performed by the same scanner that was used to acquire the mould geometry intraoperatively, the calibration process also established the transformation between the camera and robot frames, as required for the scapula-mould registration.

\subsection{Scapula-Mould Registration}

To transform the planned drill axis from the scapula's reference frame into the mould's reference frame, it is necessary for the software to know how the scapula and mould geometries fit together. The transformation between these was computed by registering the surfaces to each other. The registration transformation identified how the mould fitted onto the glenoid cavity. This allows the desired guide wire axis defined in the planning module to now be expressed in the same coordinate frame of reference as the digitised mould surface from the $3 \mathrm{D}$ optical scan. 


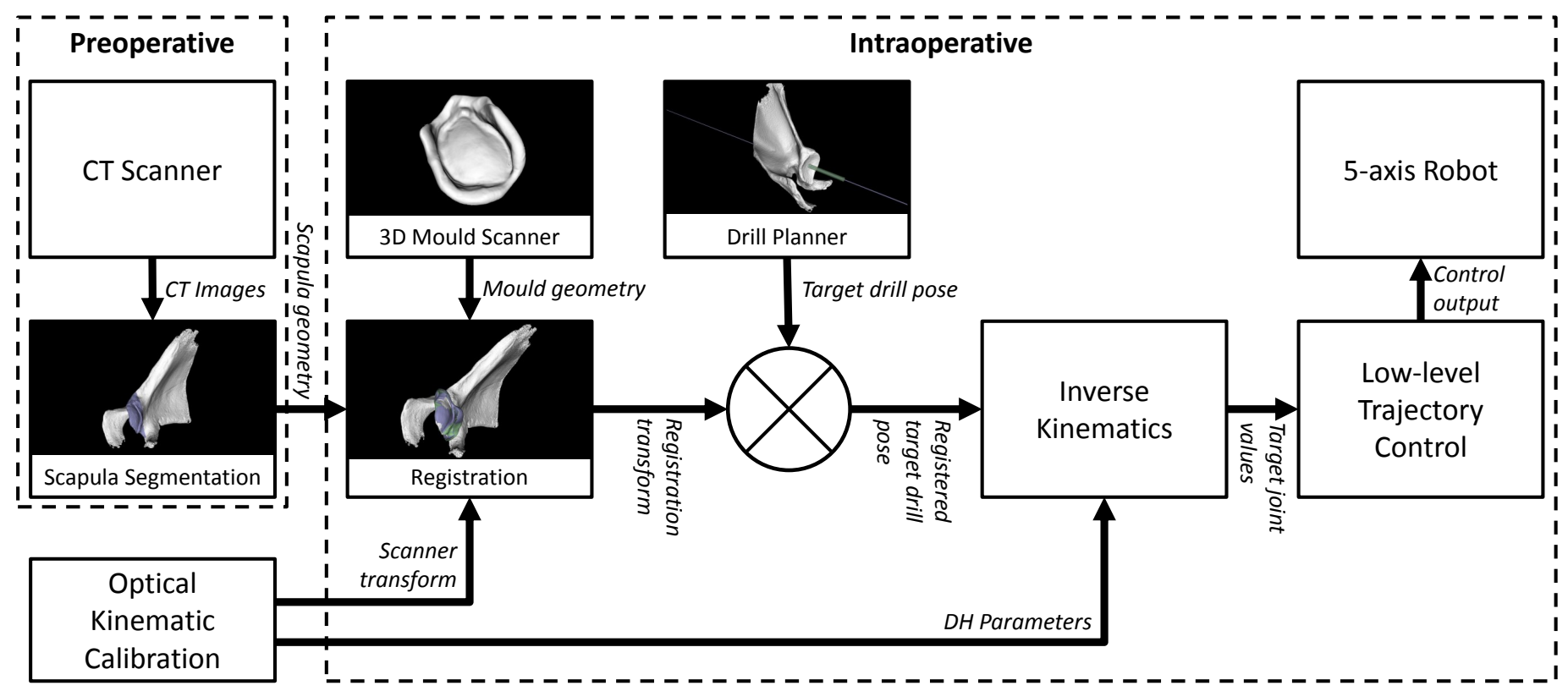

Fig. 6. Schematic diagram of the pre- and intraoperative steps of the robotic system.

The registration was performed using a variant of the Iterative Closest Point (ICP) method, ${ }^{14}$ implemented with minima suppression. ${ }^{6}$ The surgeon provided the approximate initial placement of the mould onto the scapula by matching three features (pair point matching, as in Horn ${ }^{15}$ ); however, the full ICP based registration was entirely automated. As the very edges of the mould, and hence also the scanned surface, are not in contact with the scapula, they are manually eliminated by the surgeon from the scanned surface to avoid them causing registration errors. To do this, the surgeon 'painted' the contacting surfaces in the planning software with a digital brush, such that the unpainted parts of the surface could be disregarded.

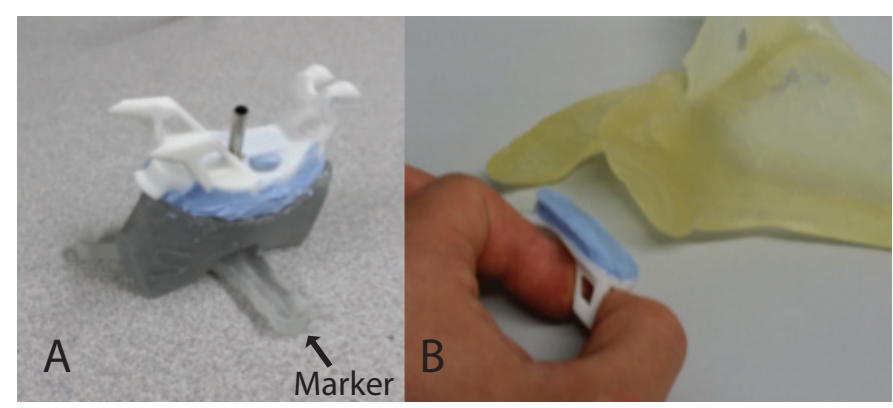

Fig. 7. A) Disposable component and guide tube placed on a synthetic glenoid with fiducial marker, in the setup used during the experiments; B) illustration of where the disposable component is placed in the real scapula

\subsection{Inverse Kinematics}

As calibrated DH parameters are used within the kinematic control of the robot, the inverse kinematics for each desired Cartesian pose were computed using convex optimisation, ${ }^{16}$ rather than analytically. Two-sided Jacobi Singular Value Decomposition (SVD), as implemented in the Eigen C++ library, ${ }^{17}$ was employed and the inverse kinematics were solved for each Cartesian pose in less than $1 \mathrm{~ms}$.

\section{Experimental Design}

We evaluated the feasibility of the approach in terms of manufacturing time and usability (intended as the ability of the surgeon to follow the prescribed work-flow) by running experimental trials with a trained surgeon, who was satisfied with the execution work-flow and average drill time ( $<3 \mathrm{~min})$. Ten anonymised shoulder CT scans of patients requiring arthroplasty were collected and segmented to produce a 3D model of the patient anatomy. A consultant orthopaedic surgeon, with arthroplasty experience, manually planned the ideal guide wire position in each scapula using the software described in section 3.1, with the aim to place the glenoid component into an appropriate position. Three, coplanar fiducial markers (Fig 7A), were also added to each glenoid model to aid comparisons by providing a common reference frame for all measurements. Each scapulaglenoid pair was 3D printed (Objet Eden, Stratasys ,USA) to produce two groups of 10 identical models: a robotically-Guided Group (GG) and a Conventional Group (CG). The surgeon manually placed a guide wire into all glenoids in the Conventional Group, according to the bespoke plan created for each glenoid, viewable via an intraoperative screen. The prototype was then 
used in the GG group to place guide wires according to identical bespoke plans for each glenoid. The operator was required to carry out the following steps and reported straightforward device operation:

(1) Take a mould of the test model with the consumable roughly orientated with respect to the glenoid cavity

(2) Place the mould in the guide manufacturing system

(3) In software, identify, by painting, sections of the mould that were in contact with the glenoid

(4) In software, perform initial registration by selecting three corresponding features on the CT model and scanned surface

(5) In software, run the automatic, ICP-based registration process to optimally align the scan surface to the CT-derived glenoid model

(6) In software, plan the desired drill axis trajectory by manually aligning the proximal and distal ends of the drill insertion axis with the registered glenoid model

(7) Initiate the automatic drilling process to machine the mould according to the plan

(8) Remove the moulded component from the machine, place it back onto the model and drill through the guidance channel

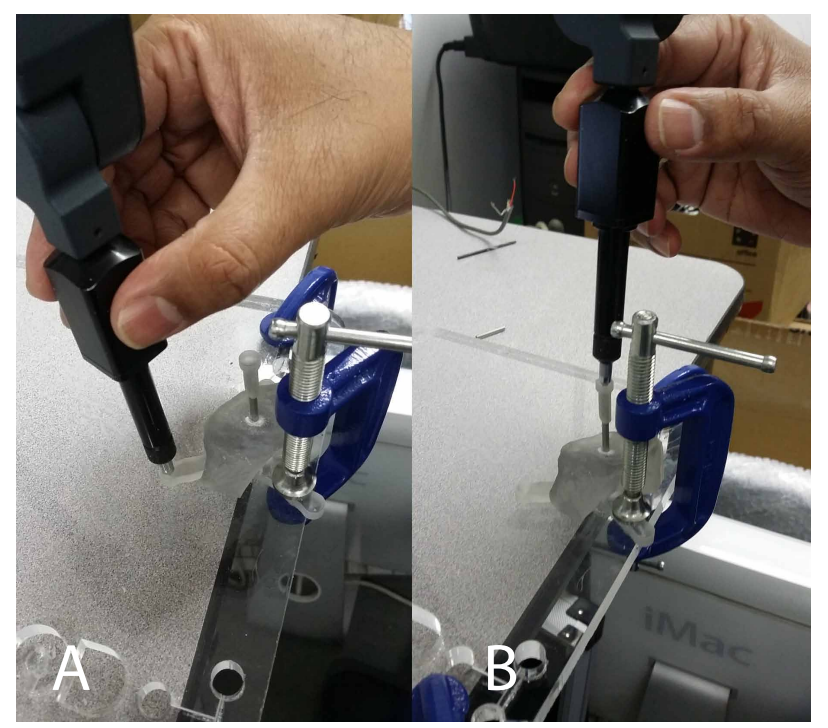

Fig. 8. Measurement of the guide placement: A) measurement of the marker position. B) measurement of position and orientation of the guide.

Once the machine had drilled the intended guide hole through each disposable, a section of $3 \mathrm{~mm}$ external diameter tubing was manually press fitted through the hole to provide drilling stability, as shown in Fig. 7. A $2.5 \mathrm{~mm}$ drill bit was used to drill through the $2.8 \mathrm{~mm}$ internal diameter tube into each glenoid model. In the manual group, the surgeon placed identical 2.5 $\mathrm{mm}$ drill bits free hand. Guide wire placement was subsequently analysed in both groups and compared to the pre-operative plan. These steps were carried out by the non-surgically trained operator after a quick initial demonstration. No technical knowledge of the underlying process was required to operate the system.

Once a guide wire was drilled into the test glenoid either manually or using our guidance system, a digitizer arm (MicroScribe G2X Digitizer, Fig. 8) was used to record the coordinates of all three conical fiducial markers in each synthetic scapula, as well as two points on the axis of the protruding wire. Once the physical points were recorded, the orientation of the wire with respect to the plane could be computed automatically. The value obtained for each sample was subsequently compared with the 'intended' axis, as described in the digital plan, to give the 'absolute' difference between achieved and intended guide wire alignment. The difference between the intended and achieved axis was measured in degrees referred to as the absolute angle. Accuracy was also expressed in both groups by comparing the Euclidean distance between the planned and achieved entry point location of the wire. This data was measured in $\mathrm{mm}$ and is referred to as the minimum distance value.

Additionally, a fully consented intraoperative moulding test was carried out to better understand the workflow integration requirements behind this step of the process. The test was carried out by a senior consultant orthopaedic surgeon at Derby Hospitals NHS trust, with an intraoperative mould of the glenoid cavity, obtained with sterilised disposable consumables, as shown in Fig.4. The surgeon provided qualitative observations as to the viability of the process and operative workflow integration.

\section{Results}

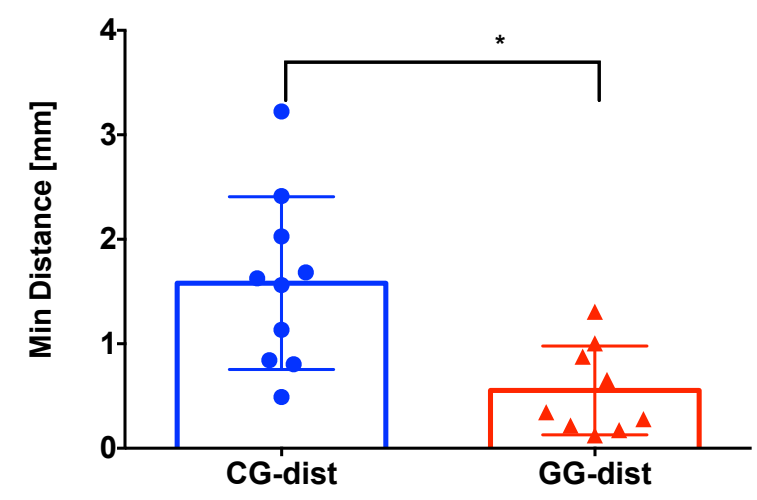

Fig. 9. Comparison of the minimal distance between the Conventional Group (CG) and the robotically Guided Group (GG). The differences are statistically significant $(\mathrm{p}=0.0183)$

Full guide manufacture was completed in approximately 5 minutes, including planning, moulding and device set up. In the Conventional Group, the average placement accuracy was $1.58 \pm 0.83 \mathrm{~mm}$ and $6.82^{\circ} \pm 4.37$ degrees, compared to the Guided Group which achieved an accuracy of $0.55 \pm 0.42 \mathrm{~mm}$ and $1.76^{\circ} \pm 0.92$ degrees. The maximum normed residual test 
(Grubb's Outlier Test with $p>0.05$ ) was used to find possible outliers in the dataset: one sample was removed from the GG (critical value $\mathrm{Z}$ : 2.289).

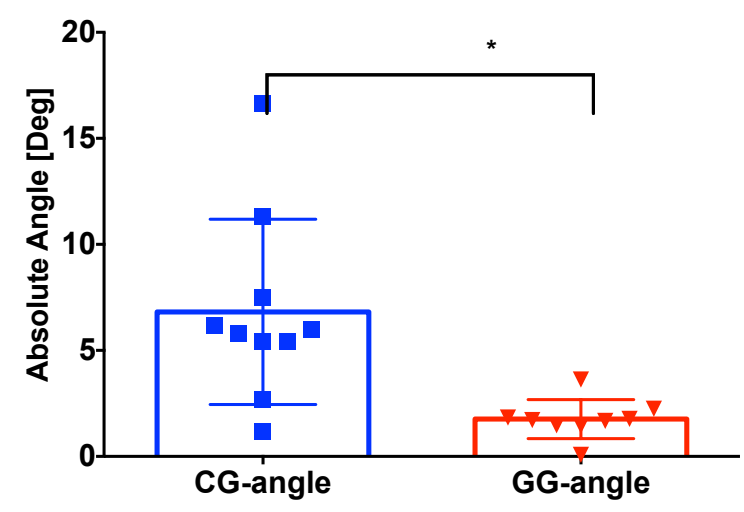

Fig. 10. Comparison of the absolute guide wire angles between the Conventional Group (CG) and the robotically Guided Group (GG). The differences are statistically significant $(\mathrm{p}=0.013)$

The normality of the datasets was checked with both Shapiro-Wilk and D'agostino-Pearson Normality test: a paired double-tail t-test (Confidence Interval 95\%) was applied to compare the minimum distance and the absolute angle metrics. The results demonstrate that the metrics were both statistically significantly affected by the method (manual vs. automatic), with: $p=0.0183$ for minimum distance and $p=0.013$ for the absolute angle. The results are graphically reported in Fig.9-10.

After the single clinical proof of concept trial in Darby, the surgeon reported the process to be straightforward and easy to integrate into the conventional work flow as "no new skills or techniques are required." The surgical process was described as "easy to explain to surgeons at all stages of training."

\section{Discussion}

Whilst the results show improved accuracy in the robotically guided group, the study may be further analysed in three distinct areas: hardware function, co-registration accuracy and surgeon accuracy. Whilst every effort was made to eliminate hardware inaccuracy through the design phase, tolerances and controller imperfections must be taken into account.

It was noted during the experiment that, whilst drilling into the test glenoids, it was difficult to hold the drill in the correct orientation. Axial instability was largely restrained by the guides and the subsequent placement of a metal sleeve into the guidance holes, however the authors are satisfied that this inaccuracy may be overcome with design modifications to the intraoperative disposables.

Reconfiguration of the intra-operative disposables allows the device and method to be used in any operative site in which a sufficient surface area of osseous anatomy is exposed and a suitable imaging volume is recorded. This paper has illustrated the example of the placement of a single guide wire in shoulder arthroplasty, however further examples may include the guided placement of two or more coplanar guide wires such that a standard cutting or drilling tool may be placed over the wires to achieve a pre-operatively defined plan.

The apparatus and method discussed in this paper offer a surgeon and the patient a number of potential advantages. As the guides are intraoperatively produced, the surgeon is in full control of guide manufacture, eliminating the logistical chain and subsequent cost associated with conventional 3D printed patient specific instrumentation. Further to this, the intraoperative workflow has the potential to be greatly simplified in comparison to existing orthopaedic intraoperative robotic solutions. The co-registration with a moulded surface has several advantages. Conventional 3D printed guides are produced from segmented image data, resulting in an often imperfect fit between the guide and patient anatomy, as the CT surface is only an estimate of the actual anatomy exposed at the time of surgery. Secondly, the surgeon can manually produce the guide from any area of exposed anatomy, provided the desired guide structures intersect the mould. This allows greater intra-operative flexibility.

\section{Conclusion}

This paper has described a novel, mechatronic system for the intraoperative manufacture of patient specific instrumentation, which was demonstrated in the context of shoulder arthroplasty under laboratory conditions. With a total guide manufacture time of 5 minutes, inexpensive consumables manufacture, a compact hardware unit and the ability to accurately place orthopaedic hardware into a preplanned position, this approach overcomes many of the drawbacks of existing orthopaedic guidance technologies. It is thus hoped that the technique may open up orthopaedic guidance so that a greater number of patients may benefit from a fully computer guided orthopaedic intervention. No direct comparison has been carried out between this approach and existing 3D printed patient specific instrumentation and orthopaedic robotics, as it was deemed to be beyond the scope of this proof of concept investigation. Whilst this remains a limitation to this validation study, the results to date demonstrate that the production and deployment of patient specific instruments in the OR is possible and can be cost effective. The placement accuracy achieved with this first prototype already surpasses manual implantation under ideal (laboratory) conditions and falls within the ballpark of many commercial offerings, with significant room for improvement. Patent WO2015075423 has been filed to protect the intellectual property underpinning this work, which is solely owned by Prometheus Surgical Ltd. as of the time of writing.

\section{References}

[1] S. Krishnan, A. Dawood, R. Richards, J. Henckel and A. Hart, A review of rapid prototyped surgical guides for patient-specific total knee replacement, Journal of Bone and Joint Surgery - British Volume 94B (2012) 1457-1461.

[2] P. F. Choong, M. M. Dowsey and J. D. Stoney, Does accurate anatomical alignment result in better function and 
quality of life? comparing conventional and computerassisted total knee arthroplasty, The Journal of Arthroplasty 24(4) (2009) 560-569.

[3] R. De Haan, P. A. Campbell, E. P. Su and K. A. De Smet, Revision of metal-on-metal resurfacing arthroplasty of the hip: The influence of malpositioning of the components, Journal of Bone and Joint Surgery, British Volume 90-B(9) (2008) 1158-1163.

[4] A. P. Skirving, Total shoulder arthroplasty current problems and possible solutions, Journal of Orthopaedic Science 4(1) (1999) 42-53.

[5] H. H. Malik, A. R. Darwood, S. Shaunak, P. Kulatilake, A. A. El-Hilly, O. Mulki and A. Baskaradas, Threedimensional printing in surgery: a review of current surgical applications, Journal of Surgical Research 199(2) (2015) $512-522$.

[6] M. Jakopec, F. Rodriguez y Baena, S. Harris, P. Gomes, J. Cobb and B. L. Davies, The hands-on orthopaedic robot "acrobot": Early clinical trials of total knee replacement surgery, Robotics and Automation, IEEE Transactions on 19 (Oct 2003) 902-911.

[7] J. Cobb, J. Henckel, P. Gomes, S. Harris, M. Jakopec, F. Rodriguez, A. Barrett and B. Davies, Hands-on robotic unicompartmental knee replacement: A prospective, randomised controlled study of the acrobot system, Journal of Bone \& Joint Surgery, British Volume 88-B(2) (2006) 188-197.

[8] J. E. Lang, S. Mannava, A. J. Floyd, M. S. Goddard, B. P. Smith, A. Mofidi, T. M. Seyler and R. H. Jinnah, Robotic systems in orthopaedic surgery, Journal of Bone and Joint Surgery - British Volume 93-B(10) (2011) 1296-1299.

[9] D. C. Beringer, J. J. Patel and K. J. Bozic, An overview of economic issues in computer-assisted total joint arthroplasty, (463) (2007) 26-30.

[10] V. Salvi, Identifit: a silicone mould used to intraoperatively construct a cementless femoral stem., La Chirurgia degli Organi di Movimento 77(4) (01/ 1992) 443-445.

[11] J. D. Slover, H. E. Rubash, H. Malchau and J. A. Bosco, Cost-effectiveness analysis of custom total knee cutting blocks, The Journal of Arthroplasty 27(2) (2012) 180 185.

[12] E. Thienpont, F. Paternostre and C. van Wymeersch, The indirect cost of patient-specific instruments., Acta Orthop. Belg 81(3) (2015) 462-470.

[13] M. Abderrahim and A. Whittaker, Kinematic model identification of industrial manipulators, Robotics and Computer-Integrated Manufacturing 16(1) (2000) 1 - 8 .

[14] P. Besl and N. D. McKay, A method for registration of 3-d shapes, Pattern Analysis and Machine Intelligence, IEEE Transactions on 14 (Feb 1992) 239-256.

[15] B. K. P. Horn, Closed-form solution of absolute orientation using unit quaternions, Journal of the Optical Society of America A 4(4) (1987) 629-642.

[16] P. I. Corke, Robotics, Vision \& Control: Fundamental Algorithms in Matlab (Springer, 2011).

[17] G. Guennebaus, B. Jacob et al., Eigen v.3 http://eigen.tuxfamily.org, (2010). 